\title{
Internet and Reference Services: Implications for Academic Libraries
}

\author{
Carolyn Radcliff \\ Mary Du Mont \\ Jeffrey Gatten
}

\begin{abstract}
Awareness and availability of information resources on the Internet, the electronic network of networks, have soared in recent years. In the process of considering the potential benefits and costs of using the Internet to access library catalogues, academic libraries discovered the existence of a new world of scholarly communication and remote information access. To take advantage of the valuable resources that are available, primarily through the efforts of research institutions and mostly in the United States, many university and college librarians are now addressing the issues of connecting to the Internet: learning to use the network, identifying resources, and educating and training users.
\end{abstract}

\section{Environment}

During the summer of 1989, Kent State University entered into a contractual agreement with Case Western Reserve University (CWRU) to participate in a telecommunications networking project. CWRU had received a substantial grant to upgrade its campus network environment to a state-of-the-art system. Kent and six other Northeast Ohio academic libraries agreed to make their online catalogues available to CWRU and to each other on the Internet with hardware provided through the grant. Kent received six IBM 3164 terminals which were installed in the libraries' reference centre.

The ability to search the online catalogues of many of the Northeast Ohio academic libraries was the original benefit of multiple institution participation in the grant project. While the hardware was being installed, however, more libraries across the United States were making their catalogues accessible over the Internet, presenting additional opportunities for resource sharing.

In preparing for public use of the Internet through dedicated terminals, the true significance of Internet access becomes apparent. Patrons can use these terminals as gateways to a vast number of resources. In order to provide guidance, librarians must investigate an unknown, intangible world of information outside the traditional library environment. In addition, a librarian needs to incorporate what is discovered into the structured services (e.g. informing, educating, providing access) for patrons. The technological complexity of Internet requires that librarians be prepared to sell its advantages to patrons, especially those who already suffer from technostress. How would one explain the Internet to a patron who has persistent difficulty in understanding the difference between a CD-ROM index and the library's online catalogue? On the other hand, many students and scholars would jump at the opportunity to use these newly available resources. 
At Kent, a networks committee was established whose goals were clear but whose tasks were vague at best, beginning with self-education. Information was pulled together from articles and other documentation, and from a committee member who had considerable experience and could act as a resource for the committee and the department. The most important part of the learning process (and the most time-consuming) was individual experimentation and investigation including subscribing to discussion lists in a variety of subject areas.

The first result was a list of the major types of resources available through the Internet: library catalogues; interest groups (electronic discussion fora); electronic journals and newsletters; fulltext databases and data archives; bibliographic indexes; guides and directories; software; and other resources. The second result was the identification of major issues.

Issues

Key issues in providing library service for Internet resources include:

- How much responsibility should librarians assume for identifying and monitoring resources on the Internet? Locally, it is difficult to find the time to devote to becoming familiar with Internet resources. Internationally, there are numerous reference tools available on electronic networks, many of them geared towards specific subject areas. Subject-specific interest groups allow subscribers to keep track of new electronic journals, full-text files, new discussion lists, and other sources in the same or related subject areas. These subject-specific interest groups are excellent for researchers and scholars who want to learn about network resources in their subject areas. The libraryoriented discussion lists provide announcements about guides to the Internet and ongoing projects to help people explore the networks. Also, librarians have begun to develop electronic and print tools which address this problem. ${ }^{1}$ These and the library-oriented discussion lists are more general reference tools.

Successful patron use of Internet resources can occur when librarians and patrons share an interest and a responsibility for effective use of Internet resources. Librarians need to empower users with necessary tools and skills while users take responsibility for determining the usefulness of resources, particularly interest groups and discussion lists.

- How can librarians learn to incorporate these new resources into traditional reference service? Communication on the networks can be frustratingly slow. Links to remote mainframe computers can go down, decreasing the certainty of a connection. Internet addresses change. These factors are undesirable at a busy reference desk, and could make librarians hesitate to use the Internet as a first source of information.

- How should librarians identify the patrons who will be served with this new resource? Faculty, graduate students, undergraduate students, and nearby communities, all stand to benefit from the information accessible through the Internet, but will come from very different perspectives. Librarians should know as much about their audiences' needs as possible in order to match them with appropriate resources. 
It may also be necessary to sell this new resource to some patrons. Because learning to access and use resources on the Internet requires a substantial investment of time and effort, allowing new users to see an immediate value can be a powerful aid. One possible solution is for library subject specialists to recommend potential discussion lists or interest groups which speak to individual interests. For example, Shakespeare scholars will find a Shakespeare discussion list and a full-text database of his plays and sonnets at Dartmouth College. Involving subject specialists requires a high degree of interest and commitment on their part.

- What is the role of the computer services department in user education? Clearly, computer services departments provide the technological foundation for Internet access on a campus. However, using resources on the Internet requires some knowledge of both network and information structures. Who provides that knowledge? One solution is for computer centres and reference departments to work together to teach users. Computer centre staff might become advisers in the use of their facilities, explaining, for example, how patrons can manage and manipulate data which they receive over the Internet.

Even as librarians become more knowledgeable about the technical aspects of networks and are able to assume more user education responsibilities, the working relationship with computer centres must remain strong. Librarians and computer centre staff should be prepared to work together to facilitate Internet access for the academic community, including, for example, the integration of Internet access with the library's online catalogue and the development of user-friendly menus and gateways.

- What is the best approach to learning to manage this new environment? Clearly, library administration support is a prerequisite for success in integrating network resources into current library services. At the reference services level, each librarian could be responsible for monitoring resources in their subject area and adding it to their subject expertise, working with the faculty, graduate students and other interested patrons. Another possibility is for one or two librarians to become the "experts" on the electronic networks and be responsible for passing on the information to patrons and other librarians. It is also useful to know that there are many librarians across the country who are familiar with Internet resources and associated issues. In addition to personal contacts, a good starting place is the electronic discussion list for reference librarianship, LIBREF-L. ${ }^{2}$

\section{Conclusion}

The Internet presents a new challenge to reference service. The profession must develop a model that incorporates the provision of access to a diversity of remote information resources, the creation of innovative services for delivering the information to users, and management of the associated costs. ${ }^{3}$ The issues of reference services and user education consume our immediate attention, but questions regarding the acquisition of electronic journals, the local archiving of retrieved data, and the provision of bibliographic access through the local library catalogue to resources available on the Internet need to be incorporated into a model of library services for the new age of scholarly communication. 


\footnotetext{
${ }^{1}$ See, for example, the Directory of Electronic Journals, Newsletters and Academic Discussion Lists, Association of Research Libraries, Washington, DC, 1991, and Library Resources on the Internet: Strategies for Selection and Use, American Library Association, Machine-Assisted Reference Section, Direct Patron Access to Computer-based Reference Systems Committee, Chicago, IL, 1991.

${ }^{2}$ Subscribe to LIBREF-L from a BITNET account by sending a message to LISTSERV@KENTVM with no subject line and the only text: "sub libref-I <your name>". (Omit the quotation marks!) If you have an Internet address, send the same message to listserv@kentvm.kent.edu. Contact the authors for further information.

${ }^{3}$ For a discussion of the larger issues surrounding the Internet and the National Research and Education Network (NREN), see West, R.P., "NREN Issues: Funding, Equity, Innovation”, Association of Research Libraries, Vol. 155, 22 March 1991, pp. 1-3.
}

\section{Further Reading}

The following selected articles are offered to provide the reader with an introductory overview of the Internet.

Alberico, R. "The Development of an 'Information Superhighway", Computers in Libraries, Vol. 10, January 1990, pp. 33-5. Describes emerging global and research networks and their potential for creating physically dispersed communities of scholars. Focuses on the National Research and Education Network (NREN), the "information superhighway". States the NREN will be a co-operative venture of government, academia, and industry that will link thousands of campuses and research labs. Speculates on a future consisting of a high-capacity network that integrates text, image, and video. Outlines the phased-implementation plans for NREN.

Arms, C.R., “A New Information Infrastructure”, Online, Vol. 14, September 1990, pp. 15-22. Explains the details of a "network" and communicates the concept of the Internet as a "network of networks". Details the history of the Internet from 1969 to present. Also relates the history of BITNET and the "convergence of BITNET and the Internet" or lack thereof. Presents the pros and cons of the decentralized administrative nature of the networks. Finally, the National Research and Education Network (NREN) is discussed as is the participation of libraries in the NREN.

Arms, C.R., "Using the National Networks: Bitnet and the Internet", Online, Vol. 14, September 1990, pp. 24-9. Details the sequential procedures to begin exploring the networks. Recommends that one first begin by using electronic mail. Once this task is mastered, electronic bulletin boards, including discussion lists, are offered as a next logical step. The next level of sophistication proposed is searching bulletin boards' archives followed by file transfer or downloading. Finally, logging into remote systems over the Internet is described. The concepts of online publications and transparent access to remote files by locally mounted software are introduced as tools for a "scholar's workstation". 
Britten, W.A., "BITNET and the Internet: Scholarly Networks for Librarians", College \& Research Libraries News, Vol. 51, February 1990, pp. 103-7. Offers practical suggestions for mastering these two communications networks. Also contains a section titled "Networkography" which lists and describes documents and services designed to lead one to references for further inquiry into the different types of resources available on the networks.

Farley, L. (Ed.), Library Resources on the Internet: Strategies for Selection and Use, RASD Occasional Papers, No. 12, American Library Association/Reference and Adult Services Division, Chicago, IL, 1992. Provides readers with an overview of the background and purpose of the Internet and of the basic commands and protocols needed to use it. Also offers strategies for identifying and using library catalogues accessible through the Internet. Includes search sheets for most major OPAC systems, and lists sources of additional information.

Kalin, S.W., and Tennant, R., "Beyond OPACS... The Wealth of Information Resources on the Internet", Database, Vol. 14, August 1991, pp. 28-33. Raises many of the same issues concerning the problems associated with identifying, evaluating and accessing the resources available on the Internet. The support and training issue is covered here as well. Also discusses the importance of ethical behaviour when one is on the Internet. Provides a sidebar on "specialized resources" that lists and describes regional information, databases in the humanities, sciences, and social sciences, and software archives.

Kesselman, M., "The Internet”, Wilson Library Bulletin, March 1992, pp. 76-8. Discusses the features and three major functions supported by Internet protocols: SMTP (Simple Mail Transfer Protocol), Telnet (remote login), and FTP (File Transfer Protocol). Provides some options for non-academics who wish to obtain access to the Internet. Concludes with a listing of sample Internet Resources and gateways.

Lynch, C.A., and Preston, C.M., "Internet Access to Information Resources", Annual Review of Information Science and Technology, Vol. 25, 1990, pp. 263-312. Serves as an extensive overview of the history and development of the Internet within the United States higher education and research community to the late 1980s. Addresses problems of locating electronic information resources, varying access interfaces and protocols, and moving information from system to system. Reports on the technologies required for information access over networks, including prototypes and development efforts. Elaborates on the development of the National Research and Education Network. Concludes with a lengthy bibliography.

Mitchell, M. and Saunders, L.M., "The Virtual Library: An Agenda for the 1990s", Computers in Libraries, Vol. 11April 1991, pp. 8, 10, 11. Attempts to "enumerate some of the issues and questions that must be resolved in order to make the virtual library a thriving enterprise".

Broaches the administrative, human and technical issues of establishing and maintaining access to networks. Calls for more collaborative planning and budgeting among libraries and computer centres. Acknowledges that electronic information copyright issues and funding will deter rapid implementation of the virtual library. 
Nickerson, G., "The Internet”, Computers in Libraries, Vol. 11, September 1991, pp. 25-9. Provides an introductory overview of the Internet, including the hierarchical organization, benefits of use, methods of access, and common problems that one might encounter. Includes illustrations of the NSFnet T1 Backbone (1990), the PACS-L Conference, and the LibSoft Archives. This article was the first in a series that appear under the title "Networked Resources".

Nielson, B., "Finding It on the Internet: The Next Challenge for Librarianship", Database, Vol. 13, October 1990, pp. 105-7. Calls for a "reinvention" of librarianship in order to accommodate electronic communications as "a major means of knowledge dissemination". Discusses the issues raised at the first organizational meeting of the Coalition for Network Information and the clues therein for reinventing librarianship. Identifying, formating, searching and retrieving resources will depend upon standards, something that librarians understand well and which they have experience. Problems with cataloguing electronic information are elaborated and ad hoc solutions that have emerged are presented. 